\title{
Angiotomografía de las Variantes Anatómicas de la Arteria Cística. Estudio en el Preoperatorio de Colelitiasis
}

\author{
Angiotomography of the Anatomical Variants of the Cystic Artery. \\ Pre-operative Study of Cholelithiasis
}

Rafael Coello Cuntó1; Ramón Roberto Gómez Araujo²; Rafael Gabriel Coello Salguero ${ }^{3}$ \& Mariano del Sol ${ }^{1,4}$

COELlO, C. R.; GÓMEZ, A. R. R.; COELLO, S. R. G. \& DEL SOL, M. Angiotomografía de las variantes anatómicas de la arteria cística. Estudio en el preoperatorio de colelitiasis. Int. J. Morphol., 37(4):1456-1462, 2019.

RESUMEN: La colecistectomía laparoscópica es el tratamiento indicado en la colelitiasis, sin embargo el procedimiento no está exento de complicaciones o morbilidad concomitante. Es posible que, debido a lesiones ductales colaterales, ocurra sangrado con posibilidad de conversión de la cirugía e indeseables resultados. Para un correcto abordaje de la región se hace fundamental la identificación del trígono cistohepático (TCH) y sus componentes, a su vez de la ligadura y sección de la arteria cística (AC). Conociendo la elevada variabilidad de la AC, el objetivo de este trabajo consistió en identificar el número, origen, trayecto y relación de la AC con el TCH y sus variaciones, utilizando angiotomografía por medio de un tomógrafo detector de 64 cortes, en el preoperatorio de 30 pacientes de sexo femenino, entre 24 y 54 años de edad, con colelitiasis diagnosticadas clínicamente y por ecosonografía. La AC en el 76,67 \% era única y se encontraba dentro del TCH, en el 16,67 \% era única y se observó fuera del TCH. En el 6,67 \% se observaron dos AC, una dentro y otra fuera del TCH. En el 66,67\% de los casos la AC se originaba de manera normal de la arteria hepática derecha. La trazabilidad de la AC fue en el 53,3\% medianamente visible y en el 46,7 \% de trazabilidad excelente. En conclusión, la identificación de la AC y sus variaciones anatómicas se puede determinar en el preoperatorio y puede ser útil para mejorar el plan quirúrgico en pacientes con colelitiasis, brindando información al procedimiento, optimizarlo y disminuir los riesgos de eventuales complicaciones relacionados con sangrado.

PALABRAS CLAVE: Colecistectomía laparoscópica; Arteria cística; Trígono cistohepático; Angiotomografía computada; Arteria hepática derecha; Anatomía laparoscópica.

\section{INTRODUCCIÓN}

La colecistectomía laparoscópica (CL) es el procedimiento aceptado para tratar la colelitiasis por su efectividad, baja incidencia de complicaciones, escaso dolor, poca estancia hospitalaria, pronto reintegro a las labores y beneficios estéticos (Scott-Conner \& Hall, 1992; Acevedo et al., 2006; Ding et al., 2007; Sugita et al., 2008; Al Helli et al., 2011).

El trígono cistohepático $(\mathrm{TCH})$ es un espacio subhepático que permite identificar al conducto cístico y a la arteria cística (AC) (Sugita et al.; Al Helli et al; Ahmed \& Sylvia, 2015); El TCH (conocido también como triángulo de la vías biliares) es prácticamente triangular formado entre el conducto cístico, el conducto hepático común y la superficie inferior del segmento V del hígado. Contiene generalmente la parte de la arteria cística próxima a la vesícula biliar, una o dos venas císticas, el linfonodo cístico y los linfáticos que salen de la vesícula, además de pequeños nervios autónomos que se dirigen a la vesícula biliar y tejido adiposo en pequeña cantidad. Por la existencia de variaciones anatómicas el TCH puede también contener conductos accesorios que drenan del hígado para la vesícula biliar.

La AC es la principal fuente de irrigación de la vesícula biliar que se localiza en la parte alta del TCH (Sugita et al; Al -Sayingh, 2010; Ahmed \& Sylvia) originándose habitualmente de la arteria hepática derecha (AHD) en distintos porcentajes (Ding et al.; Ahmed \& Sylvia; Andall et al., 2016); las variaciones del TCH llegan al 20-50\% (Balija et al., 1999; Al-Sayigh). Las presentaciones anatómicas de la AC son impredecibles y tienen una variabilidad significativa en disección laparoscópica con las consecuentes imprecisiones provocadas por la tracción y presencia de

${ }^{1}$ Programa de Doctorado en Ciencias Morfológicas, Universidad de La Frontera, Chile.

${ }^{2}$ Licenciado en Imagenología del Hospital de Especialidades Teodoro Maldonado Carbo -IESS, Guayaquil, Ecuador.

${ }^{3}$ Especialista en Gineco-Obstetricia, del Hospital de Especialidades Teodoro Maldonado Carbo -IESS, Guayaquil, Ecuador.

${ }^{4}$ Centro de Excelencia en Estudios Morfológicos y Quirúrgicos, Universidad de La Frontera, Chile. 
tejido adiposo circundante ( $8 \%$ ) (Scott-Conner \& Hall); las variaciones no excluyen grupos por sexo ni series étnicas (Ahmed \& Sylvia); se considera que la AC a su llegada a la vesícula biliar se divide en dos ramas: superficial y profunda; la vena cística no sigue el mismo curso de la arteria (Al Helli et al.). Los estudios anatómicos en Escuelas de Medicina son más diversos y variables (Ahmed \& Sylvia), tanto, que en ocasiones se informa el origen de la AC desde la arteria gastroduodenal (Michels, 1951). Lo anterior se debe a que su estudio se realizó básicamente en especímenes de autopsia (Suzuki, et al., 2000) lo cual es diferente a la anatomía observada en la disección cadavérica (Hugh et al., 1992; Nagral, 2005). La AC es tan variable en su disposición como las vías biliares (Al Helli et al.; Ahmed \& Sylvia) y las descripciones de los textos clásicos no siempre son útiles como guía para los procedimientos quirúrgicos (Michels). Además, es bien conocida la alta frecuencia de las colelitiasis en el sexo femenino sobre el masculino, en una relación de 5,25:1 (Al-Sayigh).

Un paso imprescindible de la CL es la disección del TCH y la identificación de la AC previa a su ligadura y sección. Reconocer e identificar la anatomía básica (Nagral) y quirúrgica del TCH con énfasis en la AC, pueden disminuir los riesgos durante la CL (Ata, 1991; Scott-Conner \& Hall; Sugita et al.; Al Helli et al.) ya que ladiferente posición anatómica de la AC provoca en ocasiones serios problemas quirúrgicos (Ding et al.; Ahmed \& Sylvia).

Suzuki et al. señalaron que el desconocimiento de las variaciones vasculares y ductales, así como, la identificación inadecuada, son causa de lesiones, provocando significativa morbilidad y eventual mortalidad cobrando importancia la anatomía laparoscópica (AL) del TCH.

La visualización laparoscópica es diferente a la obtenida del procedimiento por vía abierta, siendo por tanto, necesaria una identificación de dichas variaciones desde el punto de vista laparoscópico (Balija et al.). La identificación de la AC se sustenta en clasificaciones de distintos autores (Ata; Ding et al.; Suzuki et al.; Sugita et al.) que utilizaron la AL (Qamar et al., 2014a) o imágenes tomográficas, todos con la intención de disminuir el eventual e incontrolable sangrado y evitar lesiones ductales. Los estudios que están enfocados en el transoperatorio, evidencian que se han creado algunas estrategias quirúrgicas para disminuir los riesgos de lesiones inadvertidas o sangrados inesperados (Almutairi \& Hussain, 2009), ya que una impericia en el control del sangrado de la AC aumenta el riesgo de lesiones intraoperatorias de las vías biliares u otros vasos mayores, además, la utilización de succión u otras medidas extremas pueden ser inconvenientes (Balija et al.; Sugita et al.; Torres et al., 2009).
Sugita et al., examinaron 245 pacientes, en 234 (96\%), las ACs se delinearon al menos parcialmente en imágenes de TC y el TCH como las ACs se delinearon en 223 pacientes.

Considerando lo anterior, la utilización de la TC podría adicionarse en la valoración preoperatoria de colelitiasis, no solamente para aportar al conocimiento de la anatomía regional y de sus variaciones anatómicas, sino también, para optimizar y contribuir a una cirugía más segura de la CL.

\section{MATERIAL Y MÉTODO}

Previa autorización del Hospital de Especialidades Teodoro Maldonado Carbo del Instituto Ecuatoriano de Seguridad Social (IESS) de Guayaquil-Ecuador y los consentimientos informados, se estudiaron 30 pacientes de sexo femenino, entre 24 y 54 años de edad, tomados aleatoriamente, portadoras de colelitiasis, diagnosticada por ecosonografía sin cirugías previas en el hemiabdomen superior, las cuales, cumplían las valoraciones cardiovasculares y anestésicas previas con ASA1. Las pacientes se sometieron al protocolo de diagnóstico convencional al que se añadió angiotomografía para identificar la AC, efectuada hasta un mes antes de la intervención quirúrgica. Se utilizó un tomógrafo Siemens Somatón Sensation ${ }^{\circledR}$ de 64 cortes y un inyector automático de doble cabezal Medrad para el suministro simultáneo del medio de contraste diluido en solución salina.

Procedimiento angiotomográfico: los parámetro para la adquisición de las imágenes fueron: 120 kv, 300 mAs (estos pueden variar dependiendo de la contextura del paciente), con una colimación del corte de $64 \times 0,6 \mathrm{~mm}$ con un pitch de $0,65 \mathrm{~mm}$. El paciente se colocó en decúbito supino, sobre la mesa del tomógrafo, se canalizó una vía periférica con catéter 18 Fr en la fosa cubital, de preferencia en el lado derecho. Para valorar la estructura vascular se la opacificó mediante la administración de un medio de contraste IV. La inyección se realizó mediante el inyector automático; se usó un medio de contraste de alta concentración (Iopromida 370 $\mathrm{mg} / \mathrm{ml}$ ) y el volumen utilizado fue de 1 a $1,5 \mathrm{ml} / \mathrm{kg}$; por lo tanto, este pudo variar entre $80-100 \mathrm{ml}$, que a su vez fue seguido de un bolo de solución salina al 0,9\%. El examen se inició realizando un barrido simple, previo a la inyección del contraste, en cuyas imágenes se seleccionó un segmento de la aorta donde se visualizó el origen de la arteria mesentérica superior. En esta imagen se colocó una región de interés (ROI) (por sus siglas en inglés) que permitió censar en Unidades Hounsfield (UH) la concentración del medio de contraste, durante el paso por el segmento de la arte- 
ria, proceso que se denomina bolus tracking (realce arterial) de la RI. Luego se determinó un valor para cuantificar la concentración entre 100 - $150 \mathrm{UH}$, valor que al ser medido durante el procedimiento, permitió iniciar el segundo barrido que sería la serie arterial. Esta serie, posteriormente, se reconstruyó con cortes finos $(0,75 \mathrm{~mm}$ a $1 \mathrm{~mm})$ que determinó la obtención de las imágenes para la valoración de la AC.

Los parámetros de inyección fueron: 1) inyección de prueba: $20 \mathrm{ml}$ de solución salina $0,9 \%$; 2) inyección de contraste: $90 \mathrm{ml}$ de contraste IV; 3) inyección complementaria: $40 \mathrm{ml}$ de solución salina $0,9 \%$; 4) caudal de inyección: 3,5 ml/seg; 5) presión de inyección estándar: 300 psi.

Con el paciente en apnea inspiratoria se realizó el barrido de imágenes del abdomen superior, desde los hemidiafragmas hasta el margen inferior de la silueta hepática, las adquisiciones de las imágenes se realizaron utilizando un grosor el corte de 0,6 a $1 \mathrm{~mm}$ con un incremento de reconstrucción de 0,5 mm para el procesamiento del estudio.

El algoritmo de imágenes utilizado para la demostración de la AC fue: PIM fina: proyección de intensidad máxima; PIM gruesa: proyecciones en 3D; reconstrucciones multiplanares: planos axial, coronal y sagital; volumen de representación: reconstrucciones en 3D. Las relaciones anatómicas de la AC y sus variaciones se analizaron utilizando lo descrito por Suzuki et al., quienes clasificaron la AC en tres tipos: Grupo 1: La AC está situada en el TCH. Se divide en subgrupo 1a (AC única) y el subgrupo 1b (AC doble); el Grupo 2 presenta más de un aporte sanguíneo, una AC incluida en el TCH y la otra arteria fuera del TCH y el Grupo 3 en el cual las AC(s) se encuentra(n) fuera del $\mathrm{TCH}$ y se divide en $3 \mathrm{a}$ (AC única) y $3 \mathrm{~b}$ (AC doble). Para evaluar la identificación, se estableció el índice de trazabilidad de Sugita et al., que otorga un valor de 2 (excelente visibilidad), 1 (identificación parcial) y 0 (inidentificada o pobremente identificada), esta información fue incorporada al expediente, al plan de cirugía de los pacientes y los registros visuales se incorporaron al sistema telepac para visualización universal. Todos los pacientes fueron intervenidos quirúrgicamente por especialistas en Cirugía General del Instituto Ecuatoriano de Seguridad Social (IESS) con más de 10 años de experiencia.

Las variaciones de la $\mathrm{AC}$ respecto a su origen, curso y longitud fueron visualizadas través de angiotomografía y cada caso fue evaluado según lo establecido en la clasificación de Suzuki et al. Para rastrear el origen de las ACs se utilizaron imágenes de proyección de intensidad máxima adicionales de grosor arbitrario $(1-10 \mathrm{~mm})$. La trazabilidad se estimó conforme Sugita et al., identificándose la AC en los planos coronal, sagital y axial observándose además su trayecto.
Para el análisis estadístico se utilizó Epi Info ${ }^{\mathrm{TM}}$ con valores promedio. Los parámetros técnicos de evaluación fueron los siguientes: origen, trazabilidad y relación de la $\mathrm{AC}$ con el TCH, cuando fue posible.

\section{RESULTADOS}

De acuerdo al análisis de las angiografías se pudo determinar que la $\mathrm{AC}$ en 23/30 casos, $(76,67 \%$ ) era una

Tabla I. Origen de la arteria cística en 30 pacientes adultos de sexo femenino, entre 24 y 54 años de edad, portadoras de colelitiasis diagnosticada por ecosonografía sin cirugías previas en el hemiabdomen superior.

\begin{tabular}{lcr}
\hline & Frecuencia & \% \\
\hline AHD & 20 & 66,67 \\
AHD (origen) & 1 & 3,33 \\
AHD tortuosa & 1 & 3,33 \\
AHDab & 3 & 10,00 \\
AHI & 2 & 6,67 \\
AHI-AHD & 1 & 3,33 \\
AGD & 1 & 3,33 \\
AHP & 1 & 3,33 \\
\hline Total & 30 & 100,00 \\
\hline AHD: Arteria hepática derecha; AHDab: Arteria hepática \\
derecha aberrante; AHI: Arteria hepática izquierda; AHP: \\
Arteria hepática propia. AGD: Arteria gastroduodenal.
\end{tabular}

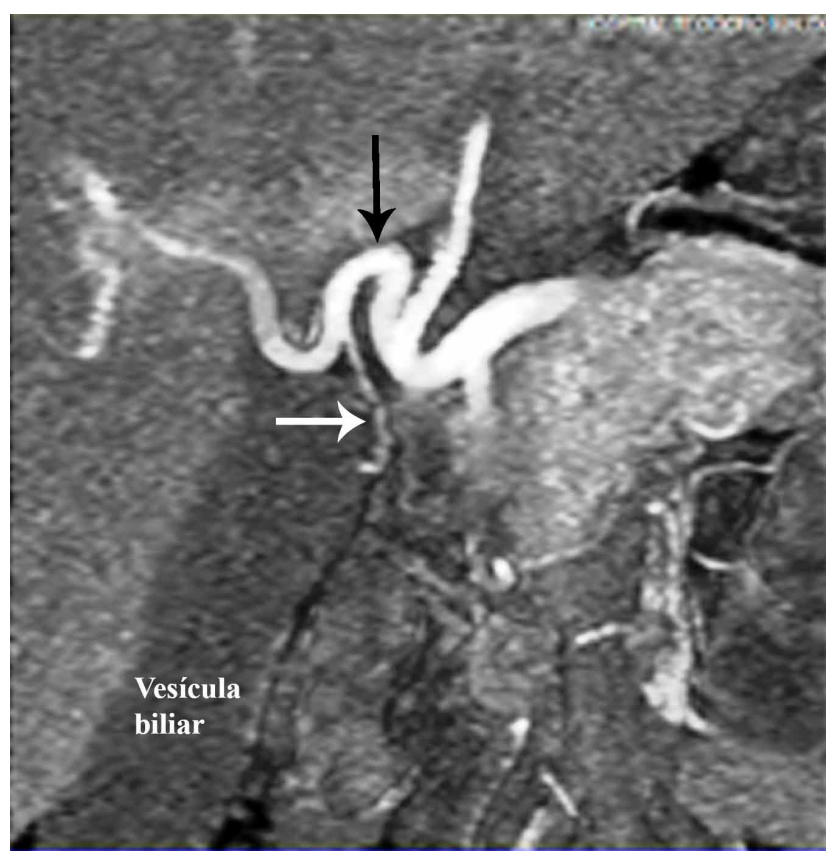

Fig. 1. ATC de paciente femenina de 57 años; arteria cística (flecha blanca) arteria hepática derecha en sifón (flecha negra). Trazabilidad: 2 . 


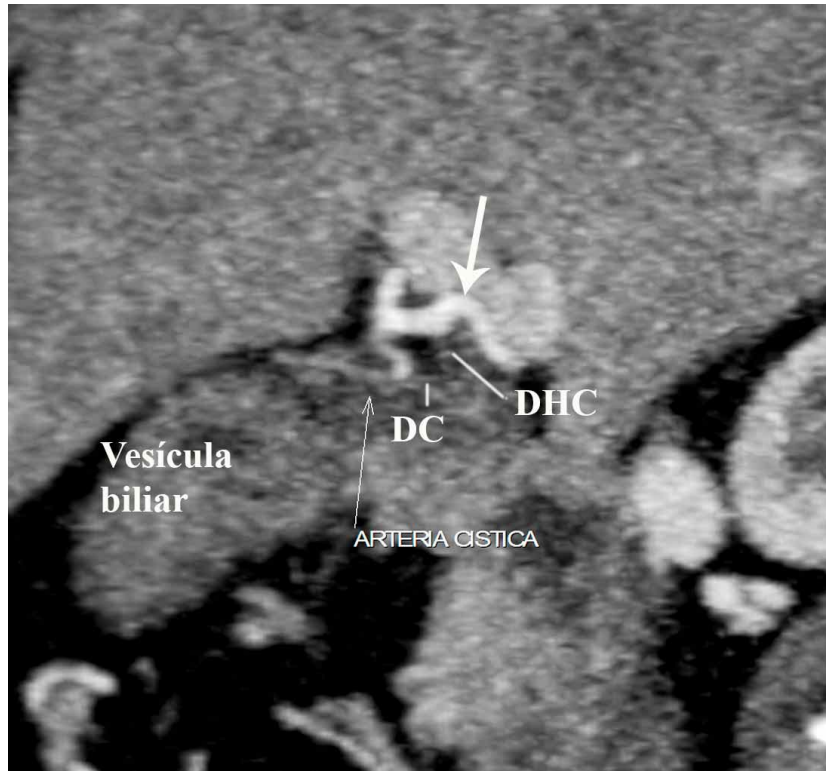

Fig. 2. ATC de pacientefemenina de 28 años; arteria hepática derecha (flecha blanca); DC: conducto cístico ; DHC: conducto hepático común. Tipo Ia y trazabilidad: 2.

Tabla II. Índice de trazabilidad de la arteria cística en 30 pacientes adultos de sexo femenino, entre 24 y 54 años de edad, a través de Angiotomografía, portadoras de colelitiasis diagnosticada por ecosonografía sin cirugías previas en el hemiabdomen superior.

\begin{tabular}{lrrcrr}
\hline Tipo & $\mathbf{1}$ & $\boldsymbol{\%}$ & $\mathbf{2}$ & \multicolumn{1}{c}{$\boldsymbol{\%}$} & \multicolumn{1}{c}{ n } \\
\hline 1a & 12 & 40 & 11 & 36,67 & 23 \\
1b & 3 & 10 & 3 & 10,00 & 6 \\
2b & 1 & 3 & 0 & 0,00 & 1 \\
\hline Total & 16 & 53 & 14 & 46,67 & 30
\end{tabular}

0: Inidentificada. 2: Excelente trazabilidad.

1: Medianamente visible.

arteria única que se encontraba dentro del TCH, en 5/30 casos $(16,67 \%)$ la $\mathrm{AC}$ era única y se visualizaba fuera del $\mathrm{TCH}$; en 2 casos $(6,67 \%)$ se observaron dos AC, una incluida en el TCH y la otra fuera TCH.

La AC se originó en 20/30 casos en la AHD normal. Variaciones en su origen fueron diversos los que pueden ser observados en la Tabla I y Figs. 1 y 2.

En el rastreo del origen de las ACs se obtuvo un 53,3 $\%$ de trazabilidad 1 (medianamente visible) y un $46,6 \%$ de trazabilidad 2 (excelente) (Tabla II, Figs.1, 2 y 3). De esta manera, la(s) AC pudieron ser seguidas y visualizadas en el $100 \%$ de los casos. Tabla II.

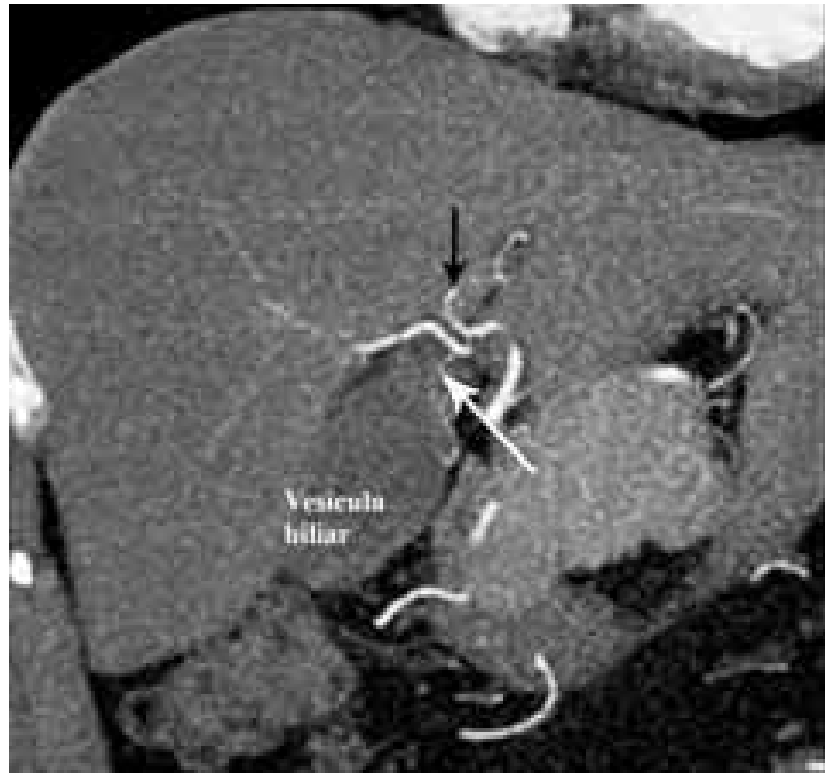

Fig. 3. ATC de paciente femenina de 51 años: arteria cística (flecha blanca); arteria hepática izquierda (flecha negra). Trazabilidad: 1 .

\section{DISCUSIÓN}

La CL se ha convertido en un procedimiento relativamente seguro y presenta muchas ventajas frente al procedimiento abierto. Las variaciones anatómicas en el TCH son frecuente en aproximadamente 20 - 50 \% según Ding et al.; Jablonska, 2008). El conocimiento de las variaciones en la anatomía arterial y ductal en relación con el TCH son decisivas durante la colecistectomía sea ésta abierta o laparoscópica (Dandekar \& Dandekar, 2016). Además, deben atenderse las variaciones de la AC debido al aumento de las intervenciones quirúrgicas del sistema biliar extrahepático (Ahmed \& Sylvia) al haberse relacionado las posibles complicaciones de la CL con la búsqueda, disección, corte y ligadura de la $\mathrm{AC}$, siendo las variaciones anatómicas del TCH quienes provocan su distorsión e inducen a errores involuntarios (Andall et al.) y malas interpretaciones.

En toda CL programada, el plan quirúrgico implica una exploración temprana del TCH para identificar precozmente la $\mathrm{AC}$, conducto cístico y conducto hepático común lo que permite minimizar complicaciones preoperatorias (Andall et al.); Es deseable establecer antes de una CL, el trayecto de la AC y su localización dentro o fuera del TCH , porque su lesión puede causar sangrado incontrolable (Sugita et al.) provocando en el mejor de los casos, solamente la conversión de la CL (Ding et al.) como recurso alternativo aunque con poca garantía por la retracción del vaso desgarrado. 
Obtener información preoperatoria detallada sobre el suministro arterial de la vesícula biliar rara vez se ha informado. Takahashi et al. (2002) establecieron una tasa de representación de AC del $90 \%$ con TC, a pesar que su objetivo era identificar otros vasos del abdomen con compromisos tumorales. Sin embargo, Sugita et al. señalaron que con el desarrollo de la TC de 64 detectores, éste ha permitido representar claramente el sistema vascular periférico y los conductos biliares extrahepáticos e identificar, al menos parcialmente, la presencia de las ACs en el $96 \%$ de los casos. Por otra parte, Wang et al. (2011) identificaron la AC con técnica contrastada, tanto para diagnóstico, como para tratamiento tumoral.

Las clasificaciones para identificar la anatomía laparoscópica de la AC son escasas; Aristotle (2014) en disecciones cadavéricas halló una AC dentro del TCH (92,5 $\%$ ) y fuera del TCH (7,5\%). Ata por AL las clasifica en 5 tipos: tipo I, tipo clásico; tipo II, AC doble identificable con magnificación del lente; tipo III, nacimiento de la AC de la AHD acodada; tipo IV, múltiples ACs y tipo V, AC aberrante. Balija et al. determinaron que la AC por vía laparoscópica se clasifica en 2 tipos: tipo I (95,5\%) dentro del TCH y tipo II $(4,55 \%)$ fuera del TCH. Ding et al. durante visiones laparoscópicas clasificaron la $\mathrm{AC}$ en tres tipos: tipo I y subtipos 1a y 1 b (AC única o doble en el $\mathrm{TCH}$ ) tipo II y subtipos (AC proveniente de varios orígenes que influye en su trayecto) y tipo III, varias ACs (ACs dentro y fuera del $\mathrm{TCH}$ ).

Sugita et al., utilizando TC con el detector de 64 cortes , también tomaron la clasificación de Suzuki et al. y

Tabla III. Variaciones de origen de la arteria cística en diferentes estudios.

\begin{tabular}{lccc}
\hline Autores & AHD \% & AHI \% & $\begin{array}{c}\text { Otros: } \\
\text { AGD-AMS \% }\end{array}$ \\
\hline Hugh \& Kelly, 1992. & $70-80$ & & \\
Balija et al., 1999. & 73,5 & 1 & 4,5 \\
Chen et al., 2000. & 41,2 & 3,9 & \\
Ding et al., 2007. & 73,3 & 3 & 7,5 \\
Ahmed \& Sylvia, 2015. & 95 & & 1,66 \\
Wang et al., 2011. & 69,5 & 4 & \\
Andall et al., 2016. & 79,02 & 2,07 & 1,94 \\
Badshah et al., 2016 & 95,5 & & \\
Scott-Conner \& Hall, 1992. & $35-75$ & & \\
Sugita et al., 2008. & 81 & 7,3 & \\
Hamza et al., 2008. & 84 & & 4,5 \\
Coello et al. & 77,33 & 6,67 & 3,33 \\
\hline
\end{tabular}

AHD: Arteria hepática derecha; AHI: Arteria hepática izquierda; AGD: Arteria gastroduodenal; AMS: Arteria mesentérica superior. obtuvieron un promedio de trazabilidad de la AC de 1,9. Por la sensibilidad y de acuerdo a Zubair et al. (2012) y Xia et al. nosotros utilizamos la clasificación de Suzuki et al. y la trazabilidad de Sugita et al. Respecto a lo anterior, nuestros hallazgos fueron: presentación 1a (76,67\%) que produjo un tiempo de disección óptimo; en aquellos con presentación 1b $(16,67 \%)$ se logró realizar procedimientos más planeados y la presentación $2 \mathrm{~b}(6,67 \%)$, permitió el abordaje con mayor precaución y detenida disección. Como es de esperar, no hubo discordancia con los hallazgos laparoscópicos, sin encontrar ningún tipo III (Tabla I).

Las variaciones de la AC son frecuentes (37\%) (Torres et al.) y se relacionan con su origen, curso y longitud (Aristotle). En nuestro estudio con 30 pacientes, se obtuvo una variabilidad de un 23,34 \%. Según Ata y AlSayigh las variaciones de la AC son más frecuentes que las ductales.

A pesar que la información preoperatoria sobre el conducto cístico puede ser crítica, concordamos con Sugita et al., en el sentido que resulta fundamental en una colecistectomía identificar a la AC.

Habitualmente la AC nace como rama de la AHD en porcentajes muy variados y está acompañada en ocasiones de un linfonodo (Qamar et al., 2014b), especialmente en presencia de procesos inflamatorios previos; en nuestro estudio la AC provenía de manera normal de la AHD en el 66,67\% sumándose aquellas que se originaban casi al nacimiento de la $\operatorname{AHD}(6,66 \%)$ y de una peligrosa aproximación de la arteria en forma de sifón (Figs. 1 y 2).

La AC puede provenir de la AHI en porcentajes menores; en nuestro estudio se originaba de esta arteria en 2 casos $(6,67 \%)$ identificándose una $\mathrm{AH}$ accesoria o arteria hepática aberrante en un $10 \%$ frente al 5,58 \% señalado por Andall et al. Otros orígenes, de la AC encontrados en nuestro trabajo fueron en la arteria gastroduodenal con un $3,33 \%$, porcentaje semejante al 4,5\% que relataron Hamsa et al.

Los autores señalados anteriormente encontraron una bifurcación clásica de la $\mathrm{AC}$ en dos ramas (61 - $87 \%$ ) y establecieron que la AC pasa delante del conducto hepático si se origina de la AHD de localización izquierda o de la AHI o detrás del conducto hepático si se origina de la arteria mesentérica superior o de la AHC.

Para rastrear el origen de las ACs Sugita et al. se utilizaron imágenes de proyección de inten- 
sidad máxima adicionales de grosor arbitrario (1-10 $\mathrm{mm})$. Se evaluó la representación clara de los vasos en toda su longitud; los casos inobservables se explicaron debido a artefactos de movimiento, grasa alrededor de la vesícula por razonables cambios inflamatorios, $\mathrm{y}$, en otros posiblemente hubo un retraso en la administración del material de contraste. La trazabilidad se estimó en un $98 \%$ (Sugita et al.) identificándose en los planos coronal, sagital y axial para definir el grado de contraste, observándose además, su trayecto, así en nuestro estudio el 53,3\% de los casos la AC presentó trazabilidad 1 (medianamente visible) y un $46,6 \%$ de trazabilidad 2 (excelente) concordando con los porcentajes de Sugita et al. (Tabla II, Figs.1-3). Coincidimos en que la TC de la AC necesita siempre una reconstrucción 3D para su confirmación, como lo mencionan Wang et al. sin olvidarse de los múltiples misceláneos en su presentación y las convalidaciones de la imagen por especialistas.

La identificación de la anatomía quirúrgica del TCH es un paso obligatorio en toda CL; han habido relativamente pocos informes sobre la AL del TCH, especialmente de la AC y dichos informes dependen de múltiples variaciones publicadas en extensas series con propósitos concomitantes; precisar las variaciones y la experiencia quirúrgica garantizan disminuir los riesgos y las eventuales complicaciones; las series muestran variaciones muy discordantes, lo que provoca un desafío en cada intervención. La ecosonografía preoperatoria no es suficiente para estos propósitos; además, la aplicación de estudios por imágenes como: colangioresonancia, CEPRE, Angiotomografía preoperatorias y la colangiografía operatoria pueden mejorar el diagnóstico y brindan un soporte para garantizar el éxito del procedimiento y sumar esta información al criterio o experiencia del equipo quirúrgico (Almutairi \& Hussain).

A pesar que los estudios por imágenes combinados como colangiografía por TC o colangiopancreatografía por RM son dirigidos a identificar las vías biliares, la ATC de 64 detectores también permite evaluar la relación entre la AC y el TCH (Sugita et al.) pero corresponden a indicaciones precisas en la actualidad, especialmente para examinar las vías biliares. Un sangrado descontrolado de la AC es un serio problema que puede aumentar el riesgo de lesión de la vía biliar, por esta razón, evaluar la AC en el preoperatorio con un estudio que garantice anticiparse ante un factor de riesgo conocido como es una lesión de la AC puede ser útil.

Con los altos índices de hallazgos de la AC por Takahashi et al. $(90 \%)$ y Xia et al. $(94 \%)$ y considerando los resultados de este estudio, se demuestra la dependencia imagenológica de la CL y su aplicación puede ser vital por las altas variaciones arteriales y ductales.
Por estas razaones, se hace necesario tener un acabado conocimiento del comportamiento normal de la AC, y por otra, es importante realizar siempre una evaluación exhaustiva de las posibles variaciones en la anatomía arterial, de los conductos hepáticos y cístico en el TCH, elementos que adquieren vital importancia durante la colecistectomía, evitando así la ligadura de manera equivocada de conductos biliares y de posibles sangramientos indeseados.

Finalmente podemos concluir que la angiotomografía, por su inocuidad y factibilidad ha demostrado ser un procedimiento practicable en instituciones que dispongan del equipamiento correspondiente; el examen presenta buena tolerancia, está exenta de riesgos mayores, brindando más seguridad en el abordaje quirúrgico; no obstante lo anterior, recomendamos realizar siempre una cuidadosa disección roma.

COELLO, C. R .; GÓMEZ, A. R. R .; COELLO, S. R. G. \& DEL SOL, M. Angiotomography of the anatomical variants of the cystic artery. Preoperative study of cholelithiasis. Int. J. Morphol., 37 (4):1456-1462, 2019.

SUMMARY: Laparoscopic cholecystectomy is the treatment indicated for cholelithiasis, however the procedure is not free of complications or concomitant morbidity. It is possible that, due to collateral ductal lesions, bleeding occurs with the possibility of surgery conversion and undesirable results. For a correct approach to the region it is essential to identify the cystohepatic trigone (CHT) and its components, as well as the ligation and section of the cystic artery (AC). Knowing the high variability of $\mathrm{CA}$, the aim of this work was to identify the number, origin, path and relationship of CA with the CHT and its variations using angiotomography by means of a 64-slice detector tomograph in the preoperative period of 30 female patients, between 24 and 54 years old, with clinically diagnosed cholelithiasis and by echo sonography. The AC in $76.67 \%$ was unique and was within the CHT, in $16.67 \%$ it was unique and was observed outside the CHT. In $6.67 \%$, two ACs were observed, one inside and one outside the TCH. In 66.67 $\%$ of cases, CA originated normally from the right hepatic artery. The traceability of AC was $53.3 \%$ moderately visible and 46.7 $\%$ excellent traceability. In conclusion, the identification of AC and its anatomical variations can be determined in the preoperative period and can be useful to improve the surgical plan in patients with cholelithiasis, providing information on the procedure, optimizing it and reducing the risks of possible bleeding related complications.

KEY WORDS: Laparoscopic cholecystectomy; Cystic artery; Cystohepatic trigone; Computed angiotomography; Right hepatic artery; Laparoscopic anatomy. 


\section{REFERENCIAS BIBLIOGRÁFICAS}

Acevedo, A. F.; Lopera, C.; Vergnaud, J. P. \& Vásquez, J. Lesiones de la vía biliar durante la colecistectomía laparoscópica Factores técnicos, anatómicos y educacionales. Rev. Colomb. Cir, 21(2):116-23, 2006.

Ahmed, M. K. \& Sylvia, S. Origin of cystic artery and its position in relation to biliary ducts and Calot's triangle. J. Evolution Med. Dent. Sci., 4(1):1$6,2015$.

Al Helli, A.; Al Taee, M. \& Al Khafaji, M. Laparoscopic surgical anatomy of Calots triangle. Kerbala J. Med., 4(9):957-63, 2011.

$\mathrm{Al}-\mathrm{Sayigh}, \mathrm{H}$. A. The incidence of cystic artery variation during laparoscopic surgery. Med. J. Babylon, 7(3-4):389-403, 2010.

Almutairi, A. F. \& Hussain, Y. A. Triangle of safety technique: a new approach to laparoscopic cholecystectomy. HPB Surg., 476159, 2009. doi: 10.1155/2009/476159

Andall, R. G.; Matusz, P.; Du Plessis, M.; Ward, R.; Tubbs, R. S. \& Loukas, M. The clinical anatomy of cystic artery variations: a review of over 9800 cases. Surg. Radiol. Anat., 38(5):529-39, 2016.

Aristotle, S. Variations in origin and course of cystic artery and its relations to Calot's triangle with its clinical implications. $O A$ Anatomy, 18:2(2):17-19, 2014.

Ata, A. H. Cystic artery identification during laparoscopic cholecystectomy J. Laparoendosc. Surg., 1(6):313-8, 1991.

Badshah, M.; Soames, R.; Nawab, J.; Baloch, F. A.; Khan, J. \& Hasnain, J. The anatomical relationship of cystic artery to Calot's triangle. J. Med. Sci., 24(4):199-201, 2016.

Balija, M.; Huis, M.; Nikolic, V. \& Stulhofer, M. Laparoscopic visualization of the cystic artery anatomy. World J. Surg., 23(7):703-7, 1999.

Chen, T. H.; Shyu, J. F.; Chen, C. H.; Ma, K. H.; Wu, C. W.; Lui, W. Y. \& Liu, J. C. Variations of the cystic artery in Chinese adults. Surg. Laparosc. Endosc. Percutan. Tech., 10(3):154-7, 2000.

Dandekar, U. \& Dandekar, K. Cystic artery: morphological study and surgical significance. Anatomy Res. Int., 2016. http://dx.doi.org/10.1155/ 2016/7201858

Ding, Y. M.; Wang, B.; Wang, W. X.; Wang, P. \& Yan, J. S. New classification of the anatomic variations of cystic artery during laparoscopic cholecystectomy. World J. Gastroenterol., WJG, 13(42):5629-34, 2007.

Hamza, M. U. Jaffar, A. A. \& Hassan, H. A. Vascular and Gallbladder Variations in Laparoscopic Cholecystectomy. Med. J. Babylon, 5(1):11934, 2008.

Hugh, T. B.; Kelly, M. D. \& Li, B. Laparoscopic anatomy of the cystic artery. Am. J. Surg., 163(6):593-5, 1992.

Jablonska, B. The arterial blood supply of the extrahepatic biliary tractsurgical aspects. Polish J. Surg., 80(6):336-42, 2008.

Michels, N. A. The hepatic, cystic and retroduodenal arteries and their relation to the biliary ducts. Ann. Surg., 133(4):503-24, 1951.

Nagral, S. Anatomy relevant to cholecystectomy. J. Minim. Access Surg., 1(2):53-8, 2005.

Qamar, N.; Ahmed, S.; Zubair, M.; Sultan, N. \& Parveen, K. Laparoscopic visualization of cystic artery variations and gender distribution in our population. Pak. J Surg., 30(1):9-13, 2014a.

Qamar, N.; Mirani, A. J.; Ahmad, S.; Parveen, K. \& Sultan, N. Lymph node significance in cystic artery identification during laparoscopic cholecystectomy. Pak. J. Surg, 30:180-2, 2014 b.

Scott-Conner, C. E. \& Hall, T. J. Variant arterial anatomy in laparoscopic cholecystectomy. Am. J. Surg., 163(6):590-2, 1992.

Suzuki, M.; Akaishi, S.; Rikiyama, T.; Naitoh, T.; Rahman, M. M. \& Matsuno, S. Laparoscopic cholecystectomy, Calot's triangle, and variations in cystic arterial supply. Surg. Endosc., 14(2):141-4, 2000.

Sugita, R.; Yamazaki, T.; Fujita, N.; Naitoh, T.; Kobari, M. \& Takahashi, S. Cystic artery and cystic duct assessment with 64-detector row CT before laparoscopic cholecystectomy. Radiology, 248(1):124-31, 2008.

Takahashi, S.; Murakami, T.; Takamura, M.; Kim, T.; Hori, M.; Narumi, Y. \& Kudo, M. Multi-detector row helical CT angiography of hepatic vessels: Depiction with dual-arterial phase acquisition during single breath hold. Radiology, 222(1):81-8, 2002.
Torres, K.; Chroscicki, A.; Golonka, A.; Torres, A.; Staskiewicz, G.; Palczak, R. \& Drop, A. The course of the cystic artery during laparoscopic cholecystectomy. Folia Morphol. (Warsz), 68(3):140-3, 2009.

Wang, X.; Shah, R. P.; Maybody, M.; Brown, K. T.; Getrajdman, G. I.; Stevenson, C. \& Solomon, S. B. Cystic artery localization with a threedimensional angiography vessel tracking system compared with conventional two-dimensional angiography. J. Vasc. Interv. Radiol., 22(10):1414-9, 2011.

Xia, J.; Zhang, Z.; He, Y.; Qu, J. \& Yang, J. Assessment and classification of cystic arteries with 64-detector row computed tomography before laparoscopic cholecystectomy. Surg. Radiol. Anat., 37(9):1027-34, 2015.

Zubair, M.; Habib, L.; Mirza, M. R.; Channa, M. A.; Yousuf, M. \& Quraishy, M. S. Anatomical variations of cystic artery: telescopic facts. Med. J. Malaysia, 67(5):494-6, 2012.

\author{
Dirección para correspondencia: \\ Dr. Mariano del Sol \\ Centro de Excelencia en Estudios Morfológicos y Quirúrgicos \\ Facultad de Medicina \\ Universidad de La Frontera \\ Temuco \\ CHILE
}

Email: mariano.delsol@ufrontera.cl

Recibido : 03-05-2019

Aceptado: 22-06-2019 